\title{
تحورات الحمض النووي الريبي \\ لجينات ndhC و ndhD وndhf البلاستيدية في أنسجة نبات \\ الوينكا
}

وسيمة بريكان الشمري ','، هشام الحربي'، هدى عطالله الحمدان'، ثناء خالد

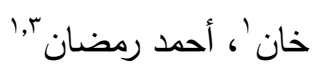

' قسم علوم الأحياء، كلية العلوم، جامعة الملك عبد العزيز بجدة، السعودية

† قسم الأحياء، كلية العلوم، جامعة حائل، حائل، السعودية الساهة

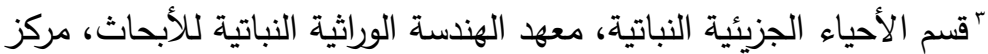

البحوث الزراعية، الجيزة، مصر

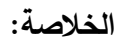

التعبير الجيني في البلاستيدات يتضمن إجراء يحدث بعد عمليه النسخ يسمى تحرير الحمض النووي

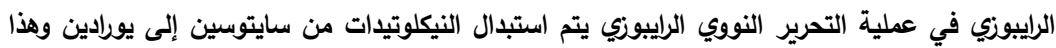

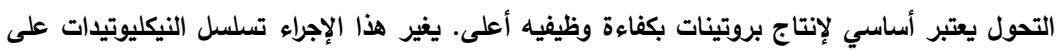

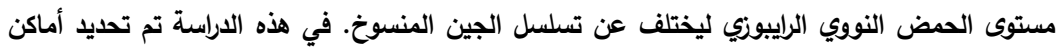

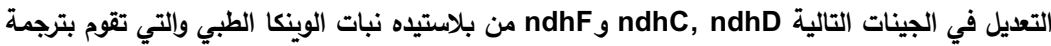



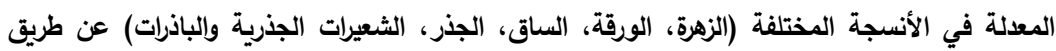

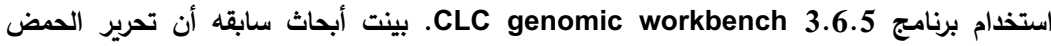



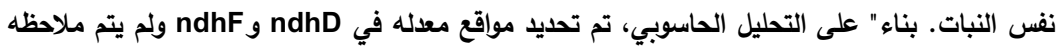

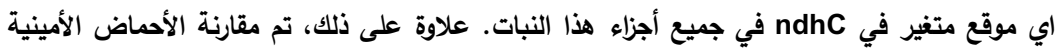

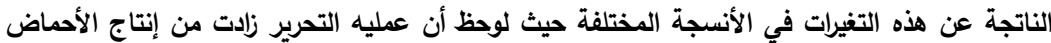
الأمينية الكارهة للماء مبينا ان هذه الإجراء قد يكون له تأثير على الجينات المستهافة. 


\section{References}

[1] Wang. W.. et al. RNA Editing in Chloronlasts of Snirodela nolvrhiza. an Aauatic Monocotelvdonous Species. PLoS One, 2015. 10(10): p. e0140285.

[2] Ichinose. M. and M. Sugita. RNA Editing and Its Molecular Mechanism in Plant Organelles. Genes (Basel), 2017. 8(1).

[3] Benne. R.. et al.. Maior transcrint of the frameshifted coxll gene from trvnanosome mitochondria contains four nucleotides that are not encoded in the DNA. cell, 1986. 46(6): $\mathrm{p}$. 819-826.

[4] Covello. P.S. and M.W. Grav. RNA editing in nlant mitochondria. Nature, 1989. 341: p. 662-666.

[5] Hoch. B.. et al.. Editing of a chloroplast mRNA bv creation of an initiation codon. Nature, 1991. 353: p. 178-180.

[6] G.Wolf. P.. C. A.Rowe. and MitsuvasuHasebe. High levels of RNA editing in a vascular plant chloronlast genome: analvsis of transcrints from the fern Adiantum capillus-veneris. Gene, 2004. 339: p. 89-97.

[7] Bock. D.K.a.R.. The Amino Acid Seauence of a Plastid Protein Is Develonmentallv Regulated bv RNA Editing. The Journal of Biological Chemistry, 2002.

[8] Yura. K. and M. Go. Correlation between amino acid residues converted bv RNA editing and functional residues in protein threedimensional structures in nlant organelles. BMC Plant Biol, 2008. 8: p. 79.

[9] Yusuke Yagi. S.H.. Keiko Kobavashi. Takashi Hiravama. Takahiro Nakamura Elucidation of the RNA Recognition Code for Pentatriconentide Reneat Proteins Involved in Organelle RNA Editing in Plants. PLOS ONE, 2013. 8(3).

[10] Okuda. K.. et al.. Pentatricopentide repeat nroteins with the DYW motif have distinct molecular functions in RNA editing and RNA cleavage in Arabidonsis chloroplasts. Plant Cell, 2009. 21(1): p. 146-56.

[11] Emi Kotera. M.T.T.S.. A pentatricopeptide reneat nrotein is essential for RNA editing in chloroplasts. Nature, 2005. 433: p. 326-330.

[12] Liere. K.. A. Weihe. and T. Börner. The transcrintion machineries of nlant mitochondria and chloroplasts: Composition. function. and regulation. Journal of Plant Physiology, 2011. 168(12): p. 1345-1360.

[13] Duff. R... and F.B.-G. Moore. Pervasive RNA Editing Among Hornwort rbcL Transcrints Excent Leiosnoroceros. Journal of Molecular Evolution, 2005. 61(5): p. 571-578.

[14] Stern. D.B.. M. Goldschmidt-Clermont. and M.R. Hanson. Chloronlast RNA Metabolism. Annual Review of Plant Biology, 2010. 61(1): p. $125-155$.

[15] Ku. C.. et al.. The Comnlete Plastid Cenome Sequence of Madagascar Periwinkle Catharanthus roseus (L.) G. Don: Plastid Genome Evolution. Molecular Marker Identification. and Phvlogenetic Imnlications in Asterids. PLoS One, 2013. 8(6): p. e68518.

[16] Kanii Ohvama. H.F.. Takavuki Kohchi. Hiromasa Shirai. Tohru Sano. Satoshi Sano, Kazuhiko Umesono, Yasuhiko Shiki,
Masavuki Takeuchi. Zhen Chang. Shin-ichi Aota. Hachiro Inokuchi \& Haruo Ozeki Chloroplast gene organization deduced from

[17] complete sequence of liverwort Marchantia nolvmornha chloroplast DNA. Nature, 1986. 322: p. 572-574.

[18] Kanii Ohvama. T.K.. Tohru Sano. Yasuvuki Yamada. Newlv identified grouns of genes in chloroplasts. Trends Biochem, 1988. 13(1): p. 19-22.

[19] Peng. L.. HiroshiYamamoto. and ToshiharuShikanai. Structure and biogenesis of the chloronlast NAD(P)H dehvdrogenase complex. Biochimica et Biophvsica Acta (BBA) - Bioenergetics, 2011. 1807(8): p. 945953.

[20] Wang. S.. et al.. Comnlete chloronlast genome seauence of Betula nlatvnhvlla: gene organization. RNA editing. and comparative and phvlogenetic analyses. BMC Genomics, 2018. 19(1): p. 950.

[21] Wang. M.. et al.. Identification and Analvsis of RNA Editing Sites in the Chloronlast Transcrints of Aegilops tauschii L. Genes (Basel), 2017. 8(1).

[22] Tillich. M.. et al.. The Evolution of Chloroplast RNA Editing. Molecular Biology and Evolution, 2006. 23(10): p. 1912-1921.

[23] Freyer, R., M.C. Kiefer-Meyer, and H. Kössel, Occurrence of plastid RNA editing in all major lineages of land plants. Proc Natl Acad Sci U S A, 1997. 94(12): p. 6285-90.

[24] Giegé. P. and A. Brennicke. RNA editing in Arabidonsis mitochondria effects $441 \mathrm{C}$ to $\mathrm{U}$ changes in ORFs. Proc Natl Acad Sci U S A, 1999. 96(26): p. 15324-9.

[25] Kalinati. Y.N.. V.D. Kumar. and S. Reddv. RNA editing of the nad3 and atn9 mitochondrial gene transcrints of Safflower (Carthamus tinctorius). International Journal of Integrative Biology 2008. 3(0973-8363): p. 143-149.

[26] Russell. M.J.B.R.B.. Amino Acid Properties and Conseauences of Substitutions, in Bioinformatics for Geneticists. 2003. 
In $n d h F$, the comparison among cDNA transcripts and $n d h F$ genome exhibited 2 editing sites in flower (nucleotide number:C1301 and C1802), 3 sites in leaf (nucleotide number: C1301, C1802, C1888), 3 sites in stem (nucleotide number: C290, C1301, C1802), 3 sites in root (nucleotide number: C290, C1301, C1802), 3 sites in hairy root (nucleotide number: (C1301, C1802, C1888) and 4 editing sites (nucleotide number: C290, C1301, C1802, C1888) were revealed in seedling Table.2 (a). As shown in table. 2 the grate number of edits presented in seedling tissue while the less edits found in flower.

All these editing sites have occurred in protein-coding regions, indicating that RNA editing is more likely to affect the structure and function of deduced protein. As a result, we have demonstrated that the $n d h D$ and $n d h F$ chloroplast transcripts of different tissues of $C$. roseus undergo to RNA editing which produce more hydrophobic amino acids residues. A previous study reported that RNA editing sites are usually found in positions important for protein formation and can convert amino acids from hydrophilic to hydrophobic form [8]. RNA editing increases the proportion of hydrophobic amino acid codons [22] and this action is important to protein and enzyme function in chloroplast like NAD(P)H dehydrogenase complex [23]. Substitution of Ser to Leu and Pro to Leu change the physicochemical properties of amino acids from hydrophilic to hydrophobic which increase the hydrophobicity of interface residues, one of the important properties of protein-protein interfaces [8]. The 4 amino acid conversions in $n d h D$ were Serine to Leucine, all of which are changing from hydrophilic to hydrophobic residues. The presence of leucine in the amino acids sequencing is essential in protein binding or recognition sites [24]. In $n d h F$, two substitutions being Serine to Leucine, one Thr to lle and one Leu to Phe. So, our result showed that four sites in $n d h D$ and two in $n d h F$ were edited to leucine in which all of these amino acids being hydrophobic. Therefore, substituted amino acids increased hydrophobicity in the deduced $n d h D$ and $n d h F$ proteins which enhances its function efficiency.

The three most frequent amino acid transitions are being Ser-Luc, Pro-Luc and Ser-Phe [22]. The deduced amino acid sequences of $n d h D$ and $n d h F$ across different organs of C.roseus after editing are converted to hydrophobic form. It is thought that these amino acids might be important for the functioning of the proteins. The amino acid Leucine is important for protein and formation of enzyme whereas phenylalanine is a common aromatic amino acid and have very hydrophobic side chains [24]. Most of the substituted amino acids due to RNA editing were hydrophobic suggesting that the hydrophobicity of deduced proteins increases their efficiency and then their function.

\section{Conclusion}

In conclusion, we identified RNA editing sites in $n d h C, n d h D$ and $n d h F$ genes (NADH-dehydrogenase subunits $3,4,5$ ) in different tissues of $C$. roseus chloroplast genome through a bioinformatics prediction soft wear. A total of five editing positions in $n d h D$ and four positions in $n d h F$, which vary depending on the tissue type, were predicted. Additionally, most of editing has led to codon modification identifying different amino acids. The most common amino acid transition in this study is being Ser-Luc. Alteration of the encoded amino acids inducing the hydrophobicity might have an influence on protein biological function. 





seedling (nucleotide number: C2, C1298, C1310) Table 1.

The initiation codon ATG was found to be created from ACG by RNA editing at $\mathrm{C} 2$ of $n d h D$ gene corresponding to the start codon in other species. Editing at $n d h D-213$ is silent, proposing that editing may no longer be required for function of protein, probably because of occurrences of compensatory mutations somewhere else in the protein complex [21]. It's obviously that stem tissues have the highest rate of editing sites with 5 edits whereas flower tissues have the lowest with only 2 edit sites.

Table: (2) RNA editing sites of $n d h F$ gene and encoded amino acids in different tissues of Catharanthus roseus

$$
\text { E: editing }
$$




hydrophobicity was increased in result of

Table: (1) RNA editing sites of $n d h D$ gene and encoded amino acids in different tissues of Catharanthus roseus E: editing

\begin{tabular}{|l|c|c|c|c|}
\hline \multirow{2}{*}{ Tissue } & \multicolumn{4}{|c|}{ Editing Sites } \\
\cline { 2 - 5 } & $\mathbf{2 9 0}$ & $\mathbf{1 3 0 1}$ & $\mathbf{1 8 0 2}$ & $\mathbf{1 8 8 8}$ \\
\hline Flower & No Editing & $\begin{array}{c}\text { E } \\
\text { Thr to IIe }\end{array}$ & $\begin{array}{c}\text { E } \\
\text { Ser to Leu }\end{array}$ & No Editing \\
\hline leaf & No Editing & $\begin{array}{c}\text { E } \\
\text { Thr to IIe }\end{array}$ & $\begin{array}{c}\text { E } \\
\text { Ser to Leu }\end{array}$ & $\begin{array}{c}\text { E } \\
\text { Leu to Phe }\end{array}$ \\
\hline Stem & $\begin{array}{c}\text { E } \\
\text { Ser to Leu }\end{array}$ & $\begin{array}{c}\text { E } \\
\text { Thr to IIe }\end{array}$ & $\begin{array}{c}\text { E } \\
\text { Ser to Leu }\end{array}$ & No Editing \\
\hline Root & $\begin{array}{c}\text { E } \\
\text { Ser to Leu }\end{array}$ & $\begin{array}{c}\text { E } \\
\text { Thr to IIe }\end{array}$ & Eer to Leu \\
Hairy root & No Editing & $\begin{array}{c}\text { E } \\
\text { Thr to IIe }\end{array}$ & $\begin{array}{c}\text { E } \\
\text { Ser to Leu }\end{array}$ & $\begin{array}{c}\text { No Editing } \\
\text { Leu to Phe }\end{array}$ \\
\hline Seedling & $\begin{array}{c}\text { E } \\
\text { Thr to IIe }\end{array}$ & $\begin{array}{c}\text { E } \\
\text { Ser to Leu }\end{array}$ & $\begin{array}{c}\text { E } \\
\text { Leu to Phe }\end{array}$ \\
\hline
\end{tabular}

a total of 4 amino acid substitutions were recognized, two being Serine to Leucine, one Threonine to Isoleucine and one Leucine to Phenylalanine, in which all being converted to hydrophobic residues.

\subsection{Analysis of the deduced amino acids sequences}

The effect of RA editing is determined at protein level. So, we compared amino acid sequences among $n d h c, n d h D$ and $n d h F$ genes and cDNA transcripts in different tissues (flower, leaf, stem, root, hairy root and seedling) of C. roseus Table 1, Fig.1.

As mention above, we reported no editing at mRNA sequences of $n d h C$ thus no amino acids change has observed. Editing status in $n d h D$ at nucleotide number 2 has been changed to methionine a start codon which important to initiate the translation process while editing at $\mathrm{C} 213$ is silent with no impact on the encoded amino acid. On the other hand, editing at nucleotide number 383, 674, 1298 and 1310 converts a Ser codon to a Leu codon. However, editing at $n d h F-290$ and 1802 modified Ser to a Leu amino acid whilst at $n d h F$ 1301 changed Thr to lle and at $n d h F-1888$ alterd Leu to phe Table 2, Fig.2. Among the 10 editing positions in both $n d h D$ and $n d h F$, the most frequently edited codon was Ser switched to Leu. As a result, the

\section{Discussion}

RNA editing is an important process that converts cytidine to uridine in higher plants chloroplasts [19]. Hence, it appears that the vital function of chloroplast RNA editing is to produce codons fundamental for protein function [20]The chloroplast $n d h C, d h D$ and $n d h F$ genes are membrane subunits encode subunit 3, 4 and5 of a plastid NAD (P) $\mathrm{H}$ dehydrogenase, a multiprotein complex involved in chloro-respiration and photosystem I [18].

We have analyzed RNA editing of $n d h C$, $n d h D$ and $n d h F$ in $C$. roseus. Editing in $n d h C$ was not reported in previous studies as well in this investigation. We found no editing event in $n d h C$ and this may indicate that $n d h C$ is available as a vital form, so it doesn't need to be edited. However, editing observed in $n d h D$ and $n d h F$, whereas the number of RNA editing sites vary among the different organs. consequently, previous studies reported that RNA editing is dependent on plant species however, in this study we demonstrated that RNA editing is dependent on tissue type as well. In $n d h D$, we identified 2 editing sites in flower (nucleotide number: C2 and C213), 4 sites in leaf (nucleotide number: C2, C383, C1298, C1310), 5 sites in stem (nucleotide number: C2, C383, C674, C1298, C1310), 4 sites in root (nucleotide number: C2, C213, C674, C1298) and 3 sites in hairy root and 
root) MK434982, (seedling) MK434983.

Transcripts of $n d h D$ under accession numbers;

MK434972 (flower), MK434973 (leaf),

MK434974 (stem), MK434975 (Root),

MK434976 (hairy root), MK434977

(seedling). Accession numbers of $n d h F$

transcripts; (flower) MK434966, (leaf)

\subsection{Predictio of $R N A$ editing in $n d h C, n d h D$ and ndhF transcripts of C.roseus}

Alignment of cDNA transcripts of different tissues of C. roseus (flower, leaf, stem, root, hairy root and seedling) revealed no editing status in

\begin{tabular}{|c|c|c|c|c|c|c|}
\hline \multirow[t]{2}{*}{ Tissue } & \multicolumn{6}{|c|}{ Editing Sites $\mathrm{C}$ to $\mathrm{T}$ and amino acid changes } \\
\hline & 2 & 213 & 383 & 674 & 1298 & 1310 \\
\hline Flower & $\begin{array}{c}\mathrm{E} \\
\text { (Thr to Met) }\end{array}$ & $\begin{array}{c}\mathrm{E} \\
\begin{array}{c}\text { No amino acid } \\
\text { change }\end{array}\end{array}$ & No Editing & No Editing & No Editing & No Editing \\
\hline leaf & $\begin{array}{c}\mathrm{E} \\
\text { (Thr to Met) }\end{array}$ & No Editing & $\begin{array}{c}\text { E } \\
\text { Ser to Leu }\end{array}$ & No Editing & $\begin{array}{c}\text { E } \\
\text { Ser to Leu }\end{array}$ & $\begin{array}{c}\text { E } \\
\text { Ser to Leu }\end{array}$ \\
\hline Stem & $\begin{array}{c}\text { E } \\
\text { (Thr to Met) }\end{array}$ & No Editing & $\begin{array}{c}\text { E } \\
\text { Ser to Leu }\end{array}$ & $\begin{array}{c}\text { E } \\
\text { Ser to Leu }\end{array}$ & $\begin{array}{c}\text { E } \\
\text { Ser to Leu }\end{array}$ & $\begin{array}{c}\mathrm{E} \\
\text { Ser to Leu }\end{array}$ \\
\hline Root & $\begin{array}{c}\mathrm{E} \\
\text { (Thr to Met) }\end{array}$ & $\begin{array}{c}E \\
\begin{array}{c}\text { No amino acid } \\
\text { change }\end{array}\end{array}$ & No Editing & $\begin{array}{c}\text { E } \\
\text { Ser to Leu }\end{array}$ & $\begin{array}{c}\text { E } \\
\text { Ser to Leu }\end{array}$ & No Editing \\
\hline Hairy root & $\begin{array}{c}\text { E } \\
\text { (Thr to Met) }\end{array}$ & No Editing & No Editing & No Editing & $\begin{array}{c}\text { E } \\
\text { Ser to Leu }\end{array}$ & $\begin{array}{c}\text { E } \\
\text { Ser to Leu }\end{array}$ \\
\hline Seedling & $\begin{array}{c}\text { E } \\
\text { (Thr to Met) }\end{array}$ & No Editing & No Editing & No Editing & $\begin{array}{c}\text { E } \\
\text { Ser to Leu }\end{array}$ & $\begin{array}{c}\text { E } \\
\text { Ser to Leu }\end{array}$ \\
\hline
\end{tabular}

MK434967, (stem) MK434968, (Root) MK434969, (hairy root) MK434970, and (seedling) MK434971.

3

4

The complete plastid genome of Madagascar Periwinkle Catharanthus.roseus was sequenced in 2013 [15]. The size of $n d h C$ is $363 \mathrm{bp}, n d h D$ is $1503 \mathrm{bp}$ and $n d h F$ is 2229 bp. Genome of $n d h C$, $n d h D$ and $n d h F$ and cDNA transcripts of flower, leaf, stem, root, hairy root and seedling tissues were downloaded and characterized using RNA-seq raw data. A total of 89,191,351 paired-end short flower RNA sequence reads, 122,063,131 paired-end short leaf RNA sequence reads, 44,283,997 paired-end short stem RNA sequence reads, 85,884,081 paired-end short root RNA sequence reads, 75,338,016 pairedend short hairy root RNA sequence reads and $74,297,533$ paired-end short seedling were mapped to the chloroplast $n d h C, n d h D$ and $n d h F$ (accession no. KC561139.1) to examine RNA events. Further, alignment across transcripts of different tissues with target genes were made to characterize the modifications at the amino acid level due to editing. $n d h C$. However, for $n d h D$ we identified $2,4,5,4$, 3,3 edits in flower, leaf, stem, root, hairy root and seedling, respectively; 2 sites in flower (nucleotide number: C2, C213), 4 sites in leaf (nucleotide number: C2, C383, C1298, C1310), 5 sites in stem (nucleotide number: C2, C383, C674, C1298, C1310), 4 sites in root (nucleotide number: C2, C213, C674, C1298), 3 sites in hairy root (nucleotide number: $\mathrm{C} 2, \mathrm{C} 1298, \mathrm{C} 1310$ ) and 3 sites in seedling (nucleotide number: $\mathrm{C} 2$, C1298, C1310) Table 1.

silent editing (ndhD-213) with no amino acid change (IIe to IIe) was identified as well. Four editing sites (ndhD-383, -674, -1298, -1310) caused amino acid conversion, all being Serine to Leucine, the most common amino acid substitution. Additionally, a comparison made among $n d h F$ and cDNA sequences showed 2, 3, $3,3,3$ and 4 edits in flower, leaf, stem, root, hairy root and seedling, respectively; 2 editing sites in flower (nucleotide number: $\mathrm{C} 1301$ and C1802), 3 sites in leaf (nucleotide number: C1301, C1802, C1888), 3 sites in stem (nucleotide number: C290, C1301, C1802), 3 sites in root (nucleotide number: C290, C1301, C1802), 3 sites in hairy root (nucleotide number: (C1301, C1802, C1888) and 4 editing sites in seedling (nucleotide number: C290, C1301, C1802, C1888) Table 2. Due to editing in $n d h F$ 
Pentatricopeptide repeat (PPR) proteins that are encoded in the nuclear genome play a central role in the post-transcriptional regulation of gene expression [9]. They are essential for the recognition of RNA editing sites and most these proteins are thought to localize in plants' plastid and mitochondria [10]; [11].

Chloroplasts are large organelles in plants responsible for photosynthesis and generate metabolic energy. They contain their own genome called plastome and their own gene expression machinery [12]. These organelles undergo RNA editing converting cytosine to uracil (C-to-U) and less frequently uracil to cytosine (U-to-C) ranging from hundreds editing sites in Hornwort and fern [6, 13]to about 30 to 40 editing sites in angiosperms [14]. Chloroplasts RNA editing has been reported in all land plants studied yet. It was first detected in $r p l 2$ gene in the maize plastid [5]. Many of the chloroplast genes were identified due to completely sequenced of number of plants chloroplasts. [15]. These chloroplast genes encode proteins complexes needed for cellular processes such as photorespiration, photosystem and other necessary organellar functions. NAD (P) $\mathrm{H}$ dehydrogenase (NDH) complex is one of these important complexes. It is encoded by 11 genes homologs to the subunits of mitochondrial NADH dehydrogenase (complex I) $[16,17]$ [18]. The chloroplast $n d h C, d h D$ and $n d h F$ genes are membrane subunits encode subunit 3, 4 and5 of a plastid NAD (P) $\mathrm{H}$ dehydrogenase, a multiprotein complex involved in chloro-respiration and photosystem I [18]

In this paper, we investigated RNA editing sites in $n d h C, n d h D$ and $n d h F$ transcripts from flower, leaf, stem, root, hairy root and seedling organs of $C$. roseus chloroplast plastome (accession no. KC561139.1). Further RNA editing sites were compared among the different tissues. Our results detected no editing status in ndhC and differential editing events among the six tissues in $n d h D$ and $n d h F$. All editing events in both genes $n d h D$ and $n d h F$ have changed the deduced amino acids except one was silent editing in $n d h D$; didn't alter the amino acid. In addition, bioinformatics analysis exhibited that RNA editing increases hydrophobicity of resultant amino acids.

\section{Materials and Methods}

\subsection{Selecting Database for ndhB chloroplast gene and $R N A$-seq}

Sequence of $n d h C, n d h D$ and $n d h F$ genes and RNA-Seq of $C$. roseus plant tissues (flower, leaf, stem, root, hairy root and seedling), were taken and downloaded from GeneBank at the National Center for Biotechnology Information Advances Science and Health (NCBI). Accession number are listed as follows: C.roseus chloroplast $n d h C$, $n d h D$ and $n d h F$ (KC561139.1); RNA-Seq: SRR122239 flower, SRR122251 leaf, SRR122253 stem, SRR122254 root, SRR122257 hairy root, SRR122243 seedling.

\subsection{Bioinformatics Analysis of RNA editing}

Sequences of $n d h C, n d h D$ and $n d h F$ genes and cDNA of different $C$. roseus tissues applied in this paper were analyzed through multi-sequence alignment using CLC genomic workbench 3.6.5, a software for predicting RNA edit sites (http://www.clcbio.com/products/clc-

genomics-workbench). The edit events detected in transcripts were compared among 6 different tissues.

\subsection{Analysis of the deduced amino acids sequences}

A comparison of amino acid sequences was made among cDNA transcripts of different organs (flower, leaf, stem, root, hairy root and seedling) of $C$. roseus after protein sequences have been deduced. The same software (Multi-sequences alignment using CLC genomic workbench 3.6.5) was employed to identify the effect of editing on the conversion of amino acids across different tissues. Finally, the identified amino acids substitutions were compared across the different organs.

\subsection{Accession Numbers}

Sequences data from this article have been submitted to GenBank data library under following accession numbers; transcripts of $n d h C$ (flower) MK434978, (leaf) MK434979, (stem) MK43498, (Root) MK434981, (hairy 


\title{
RNA Editing of chloroplast $n d h C, n d h D$ and $n d h F$ genes in Catharanthus roseus tissues
}

\author{
Wasimah B. Alshammari ${ }^{1,2}$, Hesham F. Alharby ${ }^{1}$, Huda A Alhamdan ${ }^{1}$, Thana K. Khan ${ }^{1}$, Ahmed \\ M. Ramadan ${ }^{1,3}$ \\ ${ }^{1}$ Biological Sciences Department, Faculty of Science, King Abdul-Aziz University, Jeddah, Saudi Arabia \\ ${ }_{2}$ Biological Sciences Department, Faculty of Science, Hail University, Hail, Saudi Arabia \\ ${ }^{3}$ Plant Molecular Biology Department, Agricultural Genetic Engineering Research Institute (AGERI), Agriculture Research Center \\ (ARC), Giza, Egypt \\ Email: alshamarywasima@yahoo.com
}

\begin{abstract}
Gene expression in plant chloroplast involves an important posttranscriptional process called RNA editing. At transcript level, RNA editing modifies cytidine-to-uridine and less from U-to-C which is an essential event to produce functional proteins. This action alters the nucleotide sequence of RNA transcript to differ from its gene sequence. In this study, we identified RNA editing in $n d h C$, $n d h D$ and $n d h F$ genes of Catharanthus roseus chloroplast which encode subunits of NAD (p) H dehydrogenase complex. Editing status were compared across cDNA transcripts of six tissues (flower, leaf, stem, root, hairy root and seedling). Through CLC genomic workbench 3.6.5 a bioinformatics prediction software. We reported that RNA editing event depends on the tissue type as it depends on plant species types. A total of $5 \mathrm{C}$-to-U editing positions were identified in $n d h D, 4$ positions in $n d h F$ and no editing event in $n d h C$. Furthermore, resultant amino acid from these edits were alignment among the six different tissues. We found that RNA editing increased hydrophobicity in $n d h D$ and $n d h F$ proteins, indicating that RNA editing might have an impact on the function of target genes.
\end{abstract}

Keywords: Catharanthus.roseus, RNA editing, chloroplast, $n d h C, n d h D, n d h F$, Hydrophobicity,

\section{INTRODUCTION}

Transcripts of mRNA are edited by single base substitutions, deletions or insertions at specific sites to produce functional mRNA through a process called RNA editing. Conversion of cytidine (C) to uridine (U) or (U) to (C) in RNA transcript to substitute the identity of nucleotides between RNA and its gene is an important post-transcription process to plastids RNA sequence of higher plants [1]; [2]). It was first identified in Trypanosoma brucei mitochondrial cytochrome oxidase (cox) subunit II gene [3]However, RNA editing in plant was first reported as a cytidine to uridine substitution in mitochondrial transcripts [4] and then was identified in chloroplasts [5] RNA editing is a mechanism that converts the amino acid residues in mRNAs or creates translation start or stop codons [6], thus, it is an important process to restore genetic information. Most of mechanism at mRNA transcript level which restore conserved amino acid residues [7]. Additionally, RNA editing leads in most cases to the conversion of hydrophilic amino acid to hydrophobic a substantial characteristic of protein-protein interfaces [8].RNA editing can affect protein functions by enhancing enzymatic activity of protein and controlling protein stabilization [8]. 